2 Araki S. Familial amyloidotic polyneuropathy in Japan. Amyloid and Amyloidosis. Amsterdam: Excerpta Media, 1980:67.

3 Isobe $\mathrm{T}$, Tomita $\mathrm{M}$, Matsumoto $\mathrm{J}$, et al. Plasma cell dyscrasia in 105 Japanese patients with systemic amyloidosis. Jap Med 1983;22:117.

4 Tomita H. Methods in taste examination. Proc XIIth ORL World Congr., Budapest, Hungarian Academy of Sciences 1981;627-31.

5 Henkin RI, Graziadei PPG, Bradley DF. The molecular basis of taste and its disorder. Ann Intern Med 1969;71:791-821.

6 Henkin RI, Schechter PJ, Hoye R, et al. Idiopathic Hypogeusia with dysgeusia, hyposmia, and dysosmia. JAMA 1971;217:434.

7 Young RR, Ashbury AK, Corbett JC, et al. Pure pandysautonomia with recovery. Brain 1975;98:613-36.

8 Keith DA. Oral feature of primary amyloidosis. Br J Oral Surg 1972;10:107-15.

Accepted 15 March 1986

\section{Sino-atrial arrest due to temporal lobe epilepsy}

Sir: Autonomic events may accompany many types of epileptic seizure. They can occur as a normal response to the stress of an attack, but may also result from seizure activity spreading to autonomic centres. Consequent alterations in heart rate or rhythm are among the possible causes of death during a seizure. ${ }^{1}$ Bradyarrhythmias have been noted less often than tachyarrhythmias and, to our knowledge, there have been only three documented cases of sino-atrial arrest, each in association with temporal lobe epilepsy. ${ }^{23}$ We here report a further case, in which the temporal lobe epilepsy was secondary to an intra-cranial tumour.

A 35 year old man was admitted to the Coronary Care Unit, Rochdale, in May 1982, having blacked out at the wheel of his car. He recovered quickly and there was no head injury, but he had two further brief losses of consciousness on the way to the Unit. During these he had a vacant stare with some jerky movements of the limbs, and the ambulanceman could not detect his pulse. Clinical examination on admission was normal, but he soon had a further attack witnessed by nursing and medical staff. He reported a sudden feeling of déja $v u$, at which time his pulse rate was unchanged at $50 / 60$ beats per minute. Over the next 15 seconds there was progressive bradycardia, leading to asystole for a further 20 seconds, accompanied by loss of consciousness and slight twitching of the limbs.
He was given intravenous atropine, his heart rate quickly returned to normal, and he developed a reactive hyperaemia as consciousness returned. He had five similar attacks over the next hour until a cardiac pacemaker was inserted. During each he had sufficient warning to remove his false teeth and notify staff.

There was no history of previous syncope but a month earlier he had been knocked out during a game of football and, a few days later, had an attack of déja vu unaccompanied by any disturbance of consciousness. Several similar attacks occurred on the day before admission.

Clinical examination and haematological and biochemical investigations were essentially normal. His ECG, between attacks, was also normal. However, his EEG recorded 6 days after admission showed an excess of irregular slow activity over the left temporal region. Over the next 48 hours he had no further attacks and the pacemaker was removed. Subsequent monitoring showed occasional second degree heart block (Wenkebach phenomenon) at night only.

After 2 weeks' observation, he was transferred to the Southern General Hospital and was later discharged taking phenytoin. However, he had further fits and had to be re-admitted 15 months later, complaining of left-sided headaches. Examination revealed

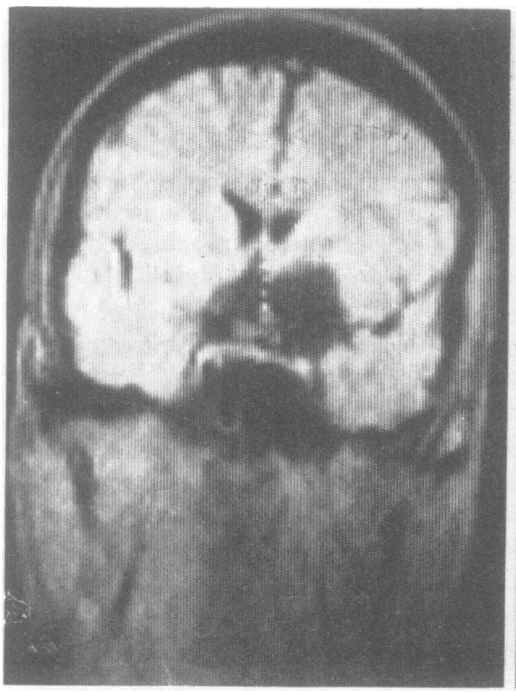

Fig Coronal and vertical sections from unclear magnetic resonance imaging of the brain. The cyst lies above the optic chiasm on both sides and extends towards the sylvian fissure and medial portion of the temporal lobe on the right.

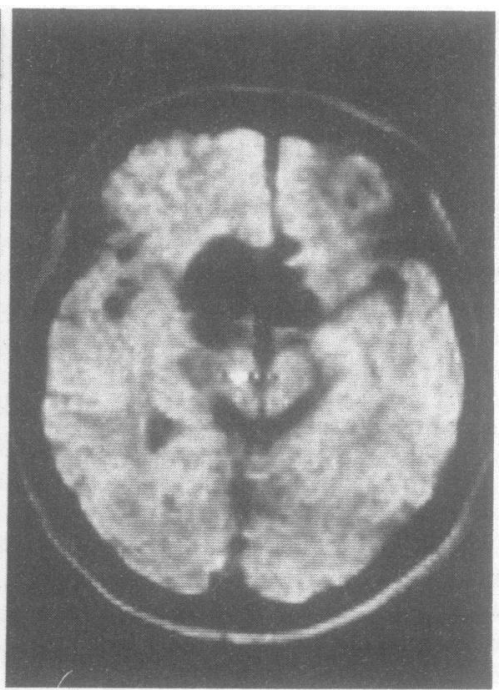

right optic atrophy and a CT scan and magnetic resonance imaging showed a suprasellar cystic lesion, thought to be a craniopharyngioma (fig).

At operation in September 1983, the lesion proved to be an epidermoid tumour adherent to the optic chiasm and undersurface of the hypothalamus and frontal lobes. The cavity was evacuated and postoperative recovery was uneventful. He has remained well and free of fits or syncope since then, with resolution of the previous visual field defect.

A recent survey of statistics concerning sudden death in epilepsy showed that in 3 to $31 \%$ of recorded cases, death was sudden, unexpected and without anatomically demonstrable cause. It was suggested that cardiac arrhythmias during a fit provide the most likely explanation for such deaths, particularly in patients with subtherapeutic anticonvulsant levels or co-existent cardiac disease. ${ }^{19}$ Furthermore, prolongation of the electrocardiographic QT interval, with its associated risk of ventricular arrhythmias may have a higher incidence among epileptic patients. ${ }^{4}$ Transitions between sleep stages and the emotional and physical stress of an attack, could act as arrhythmogenic factors through the intermediary of the autonomi system. ${ }^{15}$ In addition, ready pathways fo요을 seizure activity to directly disturb auto@ nomic function exist in the various con $\bar{\sigma}$ 
nections between cortex, limbic system, hypothalamus and brain stem, as experimental stimulation of such structures demonstrates $^{167}$

Autonomic symptoms are more frequently reported in temporal lobe epilepsy, but this may only be because consciousness is generally preserved. ${ }^{8}$ In common with other types of seizure, bradyarrhythmias are less prevalent than tachyarrhythmias. ${ }^{6-11}$ Simultaneous EEG and ECG monitoring of the present patient's attacks did not prove possible, but was achieved in two of the three previously reported instances of sinoatrial arrest during temporal lobe seizures. ${ }^{3}$ In these, asystole occurred shortly after seizure onset and lasted for 8 to 10 seconds prior to the development of a generalised convulsion. The earliest reported patient also showed abrupt asystole, lasting for up to 8 seconds, following an epigastric aura or angor animi. ${ }^{2}$ In the present case, termination of the asystolic episodes by atropine confirms that they were neurally mediated. There was no past history of simple syncope, and the onset of bradycardia was always preceded by déja vu. He had had other déja $v u$ attacks without cardiac sequelae, and was found to have temporal lobe EEG abnormalities and a tumour presumed responsible for these. We therefore feel confident that temporal lobe epilepsy was the primary cause of his asystolic episodes, and that only the subsequent loss of consciousness and jerking could have been attributable to cardiac-induced cerebral ischaemia. Whether or not the hypothalamic distortion produced by the tumour predisposed to autonomic dysfunction is not clear.

The importance of detecting a primary cardiac arrhythmia in cases of both nonepileptiform and epileptiform attacks is well established. ${ }^{1213}$ The reverse situation, that of significant cardiac arrhythmia caused by primary epileptic disturbance, may be more common than generally supposed. ${ }^{1}$ Awareness of this possibility, supported where needed by combined ECG and EEG monitoring, will help avoid one of the potentially fatal consequences of an otherwise relatively benign condition. Moreover where, as in this case, a structural lesion is responsible, it can be removed.

JOHN C SMAJE* CHRISTOPHER DAVIDSON $\dagger$ GRAHAM M TEASDALE

* North Manchester General Hospital, Manchester M86RB + Birch Hill Hospital, Rochdale OL129QB $\ddagger$ Southern General Hospital, Glasgow G5I 4TF, UK
References

1 Jay GW, Lesstma JE. Sudden death in epilepsy: a comprehensive review of the literature and proposed mechanisms. Acta Neurol Scand 1981;63, suppl 82:1-66.

2 Phizackerly PJR, Poole EW, Whitty CWM Sino-auricular heart block as an epileptic manifestation. Epilepsia 1954;3:89-91.

3 Katz RI, Tiger M, Harner RN. Epileptic cardiac arrhythmia: sino-atrial arrest in two patients. A potential cause of sudden death in epilepsy. Epilepsia 1983;24:248.

4 Rainey JM, Chayasirisobhon S. Prolongation of the QT interval in patients with epilepsy. Epilepsia 1983;24:115.

5 Johnson LC. Davidoff RA. Autonomic changes during paroxysmal EEG activity. Electroencephalogr Clin Neurophysiol 1964; 17:25-35.

6 Van Buren JM. Some autonomic concomitants of ictal automatism. Brain 1958;81:505-28.

7 Gloor P. Physiology of the limbic system. Adv Neur 1975;11:27-53.

8 Laidlaw J, Richens A, eds. A Textbook of Epilepsy. Edinburgh: Churchill Livingstone, 2nd ed, 1982.

9 Van Buren JM, Ajmone-Marsan C. A correlation of autonomic and EEG components in temporal lobe epilepsy. Arch Neurol 1960;3:683-703.

10 Pritchett ELC, McNamara JO, Gallagher JJ. Arrhythmogenic epilepsy: an hypothesis. Am Heart J 1980;100:683-8.

11 Marshall DW, Westmoreland BF, Sharbrough FW. Ictal tachycardia during temporal lobe seizures. Mayo Clin Proc 1983;58:443-6.

12 Lavy S, Stern S. Transient neurological manifestations in cardiac arrhythmias. $J$ Neurol Sci 1969;9:97-102.

13 Schott GD, McLeod AA, Jewitt PE. Cardiac arrhythmias that masquerade as epilepsy. $\mathrm{Br}$ Med J 1977;i:1454-7.

Accepted 25 January 1986

Mental status changes induced by eye drops in dementia of the Alzheimer type

Sir: Topical administration of eye drops can produce serious systemic complications in the pulmonary, cardiovascular, musculoskeletal and central nervous system. ${ }^{1-6}$ Dementia of the Alzheimer type patients are particularly vulnerable to these side effects because of the known neurochemical abnormalities that occur in this disorder. ${ }^{7}$ Recent work has demonstrated reduced activity of choline acetyltransferase and acetylcholinesterase in biopsy and post mortem brain tissues of affected patients. ${ }^{89}$ Other neuropeptides and neurotransmitters may also be significantly altered in dementia of the Alzheimer type. ${ }^{910}$ Toxic reactions from ophthalmic agents may unmask or increase the cognitive deficits, behavioural and personality changes which clinically characterise dementia of the Alzheimer type. ${ }^{7}$

Clinical reports have documented central nervous system (CNS) reactions such as confusion, hallucinations, ataxia, agitation, dysarthria and psychosis after the use of anticholinergic eye drops. ${ }^{1-13}$ Cholinomimetic agents such as pilocarpine and aceclidine are generally administered to reduce intraocular pressure in patients with certain types of glaucoma. ${ }^{14-15}$ Their clinical use has not been associated with mental changes although pilocarpine has been shown to produce complex behavioural and electrophysiological alterations in experimental animals. ${ }^{16-18}$ We report three patients who developed CNS manifestations following pilocarpine administration and were subsequently found to have dementia of the Alzheimer type.

Three elderly patients were referred to the Dementia Clinic of Thomas Jefferson University Hospital for progressive cognitive dysfunction following administration of ophthalmic drops. They had a normal medical evaluation, which included: complete blood count, sedimentation rate, thyroid, renal and liver function tests, serum Vitamin B12 and folate levels, electrolytes, urinalysis, electrocardiogram and chest radiograph. Neurological studies consisted of computed tomography (CT) of the brain, electroencephalogram (EEG) and cerebrospinal fluid (CSF) studies in all patients. Cerebral digital subtraction angiography and neuropsychological testing were done in cases 2 and 3 and magnetic resonance imaging (MRI) in case 1. All patients underwent several complete neurological examinations.

Case 1 was a 72-year-old, right-handed, white male, who presented with a 5 year history of progressive decline in short term memory and episodes of confusion and irritability, especially at night. His symptoms started after he was treated with $4 \%$ pilocarpine and $2 \%$ epinephrine eye drops for glaucoma. At that time, he also had visual hallucinations and lability of affect. He was treated with haloperidol, but this was discontinued because of tremors. His wife and daughter reported intermittent visual hallucinations and mood swings within an hour after each application of the ophthalmic medications. Neurological examination revealed an alert, elderly man, who was oriented only to person. He had no insight into his medical illness and his recent and remote memory were markedly impaired. The patient was unable to do serial $7 \mathrm{~s}$, name fingers, draw objects and follow a four-part command. He had a labile affect and right- 